\title{
Convergence of the Godunov scheme for straight line systems
}

\author{
Alberto Bressan and Helge Kristian Jenssen*
}

\section{Introduction}

Consider the Cauchy problem for an $n \times n$ system of the form

$$
u_{t}+A(u) u_{x}=0, \quad u(x, 0)=\bar{u}(x) .
$$

Here $A(u)$ is a map from a domain $\mathcal{U} \subset \mathbb{R}^{n}$ into $\mathbb{R}^{n \times n}$, and $(x, t) \in \mathbb{R} \times \mathbb{R}^{+}$. We assume strict hyperbolicity, i. e. the the matrix $A(u)$ has $n$ real and strictly different eigenvalues for each $u \in \mathcal{U}$. In the conservative case when $A(u)=D f(u)$ for some map $f: \mathcal{U} \rightarrow \mathbb{R}^{n}$, Glimm [12] proved global existence of weak entropy solutions of (1) when the data has small total variation and each characteristic field is genuinely nonlinear or linearly degenerate. The recent papers [4], [6], [7], [8] have established the uniqueness and $\mathrm{L}^{1}$ stability of solutions obtained by the Glimm scheme or by wave-front tracking. A major open question is whether other methods of approximation, such as vanishing viscosity, relaxation and finite difference schemes, yield the same solutions. In the scalar case all these methods give the unique entropy solution. Some results have been proved for $2 \times 2$ systems [11] and $n \times n$ Temple class systems [15] using compensated compactness. For general conservative systems, convergence results for vanishing viscosity approximations are known only in the case where the limit solution is piecewise smooth $[13,16]$.

In general, if one can establish a priori estimates on the total variation of approximate solutions, then Helly's theorem guarantees convergence. Using the uniqueness results in [5] one can then show that this limit is the appropriate entropy weak solution. Recently such estimates have been established for systems (1) under the assumption that the integral curves of the eigenvector fields of $A(u)$ are straight lines in state space. In [3] it was proved that vanishing viscosity yield an $\mathrm{L}^{1}$ Lipschitz continuous semigroup which is consistent with the Riemann solver. See [2], [10] for results in the case of relaxation.

In the present note we show that, under this straight-line assumption, also the approximate solutions constructed by the Godunov scheme converge to a unique limit, the same obtained by vanishing viscosity [3].

The main reason for considering these systems is that the effects of nonlinear coupling are somewhat easier to estimate. Indeed, under this straight-line

\footnotetext{
*S.I.S.S.A., Via Beirut 4, Trieste 34014, Italy, email: bressan@sissa.it, jenssen@sissa.it
} 
assumption, new oscillations can be generated only due to the interaction of waves from different families.

These particular systems were observed to be very well behaved in connection with numerical computation of slowly moving shocks. In [1] Arora and Roe conjectured that if the straight line assumption is not satisfied, then any numerical scheme using a Godunov or a Roe flux will create spurious oscillations.

Our results apply to general straight-line hyperbolic systems of the form (1), not necessarily in conservation form. For simplicity we will outline the proofs only in the conservative case. For all details we refer to [9].

\section{The Godunov Scheme and Main Results}

Let the matrix $A(u)$ in (1) have $n$ real and distinct eigenvalues $\lambda^{k}(u), k=$ $1, \ldots, n, u \in \mathcal{U}$. By a linear change in the $t$ - $x$ coordinates we can assume that

$$
0<\lambda^{1}(u)<\cdots<\lambda^{n}(u)<1 .
$$

We also assume that these characteristic speeds are uniformly separated, i.e. there exists constants $\bar{\lambda}^{0}=0<\bar{\lambda}^{1}<\ldots<\bar{\lambda}^{n-1}<\bar{\lambda}^{n}=1$ such that

$$
\left.\lambda^{k}(u) \in\right] \bar{\lambda}^{k-1}, \bar{\lambda}^{k}[, \quad u \in \mathcal{U}, \quad k=1, \ldots, n .
$$

The corresponding right and left eigenvectors are denoted by $r^{k}(u), l^{k}(u)$, respectively, and normalized such that $\left|r^{k}(u)\right| \equiv 1$ and $r^{i}(u) \cdot l^{j}(u) \equiv \delta_{i, j}$. The basic assumption is that the integral curves of the eigenvector fields are straight lines. This can be expressed by the relation

$$
\left(D r^{k}(u)\right) r^{k}(u)=0, \quad u \in \mathcal{U}, \quad k=1, \ldots, n,
$$

where $D r^{k}$ denotes the Jacobian matrix of $r^{k}$.

\subsection{The Riemann Solver}

Consider the Riemann problem for (1) with data

$$
\bar{u}(x)= \begin{cases}u_{-} & \text {for } x<0 \\ u_{+} & \text {for } x>0 .\end{cases}
$$

For $u \in \mathcal{U}, k=1, \ldots, n$, let $R^{k}(\cdot)(u)$ be the integral curve of $r^{k}$ through $u$,

$$
R^{k}(\sigma)(u)=u+\sigma r^{k}(u)
$$

Given $u_{-}, u_{+} \in \mathcal{U}$, by strict hyperbolicity there exist unique intermediate states $\omega_{0}=u_{-}, \omega_{1}, \ldots, \omega_{n}=u_{+}$and wave strengths $\sigma^{k}$ such that

$$
\omega_{k}=R^{k}\left(\sigma^{k}\right)\left(\omega_{k-1}\right), \quad k=1, \ldots, n .
$$

For each $k$, define the scalar function

$$
F^{k}(\sigma)=\int_{0}^{\sigma} \lambda^{k}\left(R^{k}(s)\left(\omega_{k-1}\right)\right) d s
$$


and let $z^{k}(x, t)$ be the unique entropy solution to the scalar Riemann problem

$$
z_{t}+F^{k}(z)_{x}=0, \quad z(x, 0)= \begin{cases}0 & \text { for } x<0 \\ \sigma^{k} & \text { for } x>0\end{cases}
$$

To solve the Riemann problem $\left(u_{-}, u_{+}\right)$we use the solution of these scalar Riemann problems to move along the integral curves. More precisely, we first define the map $\mathcal{R}\left(u_{-}, u_{+}\right):[0,1] \rightarrow \mathcal{U}$ by

$$
\mathcal{R}\left(u_{-}, u_{+}\right)(\xi)=R^{k}\left(z^{k}(\xi, 1)\right)\left(\omega_{k-1}\right) \quad \text { for } \xi \in\left[\bar{\lambda}^{k-1}, \bar{\lambda}^{k}\right], \quad k=1, \ldots, n .
$$

We then define the solution of the Riemann problem (1),(4) as

$$
u(x, t)= \begin{cases}u_{-} & \text {for } x / t<0 \\ \mathcal{R}\left(u_{-}, u_{+}\right)(x / t) & \text { for } x / t \in[0,1] \\ u_{+} & \text {for } x / t>1\end{cases}
$$

\subsection{The Godunov Scheme}

By the assumption (2) we can choose equal time and space step $\Delta x=\Delta t$. The constant value of the approximate solution in the $i$-th cell at time $j \Delta t$ is denoted by $u_{i, j}$. The Godunov scheme is now defined inductively as follows: At time $t=0$ we let $u_{i, 0}$ denote the cell average over the $i$-th cell of the initial data $\bar{u}$. Given the values $u_{i-1, j}$ and $u_{i, j}$ in the $(i-1)$-th and $i$-th cells, respectively, at time $j \Delta t$, we define the value $u_{i, j+1}$ by

$$
u_{i, j+1}=\frac{1}{\Delta x} \int_{0}^{\Delta x} \mathcal{R}\left(u_{i-1, j}, u_{i, j}\right)(x / \Delta t) d x=\int_{0}^{1} \mathcal{R}\left(u_{i-1, j}, u_{i, j}\right)(\xi) d \xi .
$$

For each $\Delta x$ the scheme gives an approximate solution $u_{\Delta x}$ of (1) defined for all $(x, t) \in \mathbb{R} \times \mathbb{R}^{+}$.

\subsection{Main Results}

We now state the main results of the paper.

Theorem 1 ( $\boldsymbol{B} \boldsymbol{V}$ bounds and $\boldsymbol{L}^{1}$ stability) Suppose that $A(u)$ is strictly hyperbolic for each $u \in \mathcal{U}$ and that the normalized eigenvectors satisfy (3). Then there exist constants $\delta_{0}, \delta_{1}>0$ such that the following holds. For each initial data $\bar{u} \in L^{1}$ with

$$
T . V \cdot[\bar{u}]<\delta_{0},
$$

the corresponding solution $u_{i, j}$ of the Godunov scheme (8) is well defined for all time steps $j \in \mathbb{Z}_{+}$and satisfies

$$
\text { T.V. }[u(j)]<\delta_{1}, \quad \text { for all } j \in \mathbb{Z}_{+} .
$$

Furthermore, there exists a constant $L$ such that for all pairs of initial data $\bar{u}, \bar{v}$ satisfying (9), the corresponding solutions satisfy

$$
\left\|u(j)-v\left(j^{\prime}\right)\right\|_{L^{1}} \leq L \cdot\left(\left|j-j^{\prime}\right| \cdot \Delta t+\|\bar{u}-\bar{v}\|_{L^{1}}\right) .
$$


Theorem 2 (Convergence) As the discretization parameter $\Delta x=\Delta t$ tends to zero, the approximate solutions given by the Godunov scheme (8) converge to the same limit as given by the method of vanishing viscosity. This limit can be characterized as the trajectory of a semigroup $S: \mathcal{D} \times \mathbb{R}^{+} \rightarrow \mathcal{D}$ with the properties:

(i) Every initial data $\bar{u} \in L^{1}$ with sufficiently small total variation lies in the domain $\mathcal{D}$ of the semigroup.

(ii) For every $\bar{u}, \bar{v} \in \mathcal{D}$ and every $t, s \geq 0$,

$$
\left\|S_{t} \bar{u}-S_{s} \bar{v}\right\|_{L^{1}} \leq L \cdot\left(|t-s|+\|\bar{u}-\bar{v}\|_{L^{1}}\right)
$$

(iii) For every piecewise constant initial data $\bar{u} \in \mathcal{D}$, there exists a positive time $\tau>0$ such that the the semigroup trajectory $S_{t} \bar{u}$ on $[0, \tau]$ coincides with the function obtained by patching together the solutions of the Riemann problems given by $\bar{u}$ and solved according to (7).

In particular, for conservative systems the Godunov scheme yields the trajectories of the Standard Riemann Semigroup.

\section{Wave strengths and wave speeds}

We treat the conservative case with $A=D f$. The Riemann solver is then the standard one and the Godunov scheme can be written in finite difference form

$$
u_{i, j+1}=u_{i, j}-\left[f\left(u_{i, j}\right)-f\left(u_{i-1, j}\right)\right], \quad i \in \mathbb{Z}, \quad j \in \mathbb{Z}_{+} .
$$

Given a left state $u_{-}$and a right state $u_{+}$connected by a single $k$-wave of strength $\sigma^{k}$, the speed of the wave is

$$
\lambda^{k}\left(u_{-}, u_{+}\right)=\int_{0}^{1} \lambda^{k}\left(u_{-}+\xi \sigma^{k} r^{k}\left(u_{-}\right)\right) d \xi .
$$

Given three consecutive states $u_{-}, u_{0}, u_{+}$, let $\bar{u}_{-}, \bar{u}_{+}$be the resulting states,

$$
\bar{u}_{-}=u_{0}-\left[f\left(u_{0}\right)-f\left(u_{-}\right)\right], \quad \bar{u}_{+}=u_{+}-\left[f\left(u_{+}\right)-f\left(u_{0}\right)\right] .
$$

The strengths of the $k$-waves, $k=1, \ldots, n$, in the three Riemann problems $\left(u_{-}, u_{0}\right),\left(u_{0}, u_{+}\right)$, and $\left(\bar{u}_{-}, \bar{u}_{+}\right)$are denoted by $\sigma_{-}^{k}, \sigma_{+}^{k}$, and $\bar{\sigma}^{k}$, respectively. The intermediate states in the two Riemann problems $\left(u_{-}, u_{0}\right)$ and $\left(u_{0}, u_{+}\right)$are denoted by $u_{-}^{1}, \ldots, u_{-}^{n-1}$ and $u_{+}^{1}, \ldots, u_{+}^{n-1}$, respectively. Now define the map

$$
\Psi^{k}\left(u_{0} ; \sigma_{-} ; \sigma_{+}\right)=\bar{\sigma}^{k}-\left(\lambda^{k}\left(u_{-}^{k-1}, u_{-}^{k}\right) \sigma_{-}^{k}+\left[1-\lambda^{k}\left(u_{+}^{k-1}, u_{+}^{k}\right)\right] \sigma_{+}^{k}\right),
$$

where $\sigma_{ \pm}=\left(\sigma_{ \pm}^{1}, \ldots, \sigma_{ \pm}^{n}\right)$. We have the following representation. 
Lemma 1 Suppose that the straight-line assumption (3) holds and let $\Psi^{k}$ be defined by (16). Then there exist smooth functions $A_{p, q}^{k}, B_{p, q}^{k}$, and $C_{p, q}^{k}$ depending on $u_{0}, \sigma_{-}, \sigma_{+}$such that

$$
\Psi^{k}\left(u_{0} ; \sigma_{-} ; \sigma_{+}\right)=\sum_{1 \leq p \neq q \leq n}\left(A_{p, q}^{k} \sigma_{+}^{p} \sigma_{+}^{q}+B_{p, q}^{k} \sigma_{+}^{p} \sigma_{-}^{q}+C_{p, q}^{k} \sigma_{-}^{p} \sigma_{-}^{q}\right)
$$

for all $u_{0} \in \mathcal{U}$ and for all sufficiently weak strengths $\sigma_{ \pm}^{1}, \ldots, \sigma_{ \pm}^{n}$.

By a representation result for smooth maps it suffices to show that $\Psi^{k}$ vanishes whenever $\sigma_{-}, \sigma_{+}$contain only one wave, of the same family. That is, $\Psi^{k}\left(u_{0} ; \sigma_{-} ; \sigma_{+}\right)=0$ whenever there exists an index $j$ such that

$$
\sigma_{+}^{p}=\sigma_{-}^{p}=0 \quad \forall p \neq j
$$

The above identity is a consequence of the definition of $\Psi^{k}$ and the assumption (3). The lemma shows that the increase in total variation is due only to transversal terms. Returning to the scheme, we let $\sigma_{i, j}^{k}$ be the strength of the $k$ wave in the Riemann problem $\left(u_{i-1, j}, u_{i, j}\right)$, and we let $\lambda_{i, j}^{k}$ be the corresponding speed given. By Lemma 1 the strengths satisfy the system

$$
\sigma_{i, j+1}^{k}=\lambda_{i-1, j}^{k} \sigma_{i-1, j}^{k}+\left(1-\lambda_{i, j}^{k}\right) \sigma_{i, j}^{k}+Q_{i, j}^{k},
$$

for $k=1, \ldots, n$, where the quadratic coupling terms are given by

$$
Q_{i, j}^{k}=\sum_{1 \leq p \neq q \leq n}\left(A_{p, q}^{k} \sigma_{i, j}^{p} \sigma_{i, j}^{q}+B_{p, q}^{k} \sigma_{i, j}^{p} \sigma_{i-1, j}^{q}+C_{p, q}^{k} \sigma_{i-1, j}^{p} \sigma_{i-1, j}^{q}\right) .
$$

\section{A priori bounds on the total variation}

We obtain the a priori bounds by bounding the total amount of waves in each family uniformly in time. That is, we establish estimates on the sums

$$
\mathrm{V}^{k}(j):=\sum_{i=-\infty}^{\infty}\left|\sigma_{i, j}^{k}\right|, \quad k=1, \ldots, n,
$$

that are independent of the time step $j$. We derive a functional relation which implies that if the initial amount of waves is sufficiently small, then it remains small for all times. The functional relation is deduced by applying Duhamel's principle, and a basic estimate on the product of two Green kernels. From (18) it follows that

$$
\mathrm{V}^{k}(j+1) \leq \mathrm{V}^{k}(j)+\sum_{i=-\infty}^{+\infty}\left|Q_{i, j}^{k}\right| \leq \mathrm{V}^{k}(0)+\sum_{m=0}^{j} \sum_{i=-\infty}^{+\infty}\left|Q_{i, m}^{k}\right| .
$$

Defining the magnitude $\mathrm{Q}^{k}(j)$ by

$$
\mathrm{Q}^{k}(j):=\sum_{m=0}^{j} \sum_{i=-\infty}^{+\infty}\left|\mathrm{Q}_{i, m}^{k}\right|
$$


and using (19), we obtain the following bound

$$
\mathrm{Q}^{k}(j) \leq C_{0} \cdot \sum_{p \neq q} \sum_{m=0}^{j} \sum_{i=-\infty}^{+\infty}\left(\left|\sigma_{i, m}^{p}\right|\left|\sigma_{i, m}^{q}\right|+\left|\sigma_{i-1, m}^{p}\right|\left|\sigma_{i, m}^{q}\right|\right)=: E_{1}+E_{2},
$$

where $C_{0}$ is a suitable constant. Let $\Gamma^{k}\left(i, j ; i^{\prime}, j^{\prime}\right)$ denote the Green kernel corresponding to the homogeneous version of (18). By Duhamel's principle we can write the solution of the non-homogeneous system (18) as

$$
\sigma_{i, j}^{k}=\sum_{l=-\infty}^{+\infty} \Gamma^{k}(i, j ; l, 0) \sigma_{l, 0}^{k}+\sum_{r=0}^{j-1} \sum_{l=-\infty}^{+\infty} \Gamma^{k}(i, j ; l, r) \mathrm{Q}_{l, r}^{k}=: \alpha_{i, j}^{k}+\beta_{i, j}^{k} .
$$

We thus have,

$$
\begin{aligned}
E_{1} \leq C_{0} \cdot \sum_{p \neq q} \sum_{m=0}^{j} \sum_{i=-\infty}^{+\infty}\left(\left|\alpha_{i, m}^{p}\right|\left|\alpha_{i, m}^{q}\right|+\left|\alpha_{i, m}^{p}\right|\left|\beta_{i, m}^{q}\right|+\right. \\
\left.\left|\beta_{i, m}^{p}\right|\left|\alpha_{i, m}^{q}\right|+\left|\beta_{i, m}^{p}\right|\left|\beta_{i, m}^{q}\right|\right) \\
=C_{0} \cdot\left(S_{1}+S_{2}+S_{3}+S_{4}\right) .
\end{aligned}
$$

To proceed we must estimate the total interaction between Green kernels.

Lemma 2 Assume that $0<\lambda_{i, j}^{p}<\lambda<\tilde{\lambda}<\lambda_{i^{\prime}, j^{\prime}}^{q}<1$ for all $(i, j),\left(i^{\prime}, j^{\prime}\right) \in$ $\mathbb{Z} \times \mathbb{Z}_{+}$. Let $\rho_{i, j}^{p}$ and $\rho_{i, j}^{q}$ denote the solutions of (18) in the homogeneous case where $Q_{i, j}^{p}=Q_{i, j}^{q} \equiv 0$ and with initial data $\rho_{0}^{p}$ and $\rho_{0}^{q}$, respectively. Then,

$$
\sum_{m=0}^{j} \sum_{i=-\infty}^{+\infty}\left|\rho_{i, m}^{p}\right|\left|\rho_{i, m}^{q}\right| \leq C(p, q) \cdot\left(\sum_{i=-\infty}^{+\infty}\left|\rho_{0}^{p}(i)\right|\right) \cdot\left(\sum_{i=-\infty}^{+\infty}\left|\rho_{0}^{q}(i)\right|\right) .
$$

To prove the result one first consider the constant coefficient case and then extends by a comparison argument. Thanks to Lemma 2, we can now estimate each term on the right hand side of (25). By (24) and (26) we have

$$
S_{1}=\sum_{p \neq q} \sum_{m=0}^{j} \sum_{i=-\infty}^{+\infty}\left|\alpha_{i, m}^{p} \| \alpha_{i, m}^{q}\right| \leq C_{1} \cdot \sum_{p \neq q} \mathrm{~V}^{p}(0) \cdot \mathrm{V}^{q}(0)
$$

where $C_{1}:=\max _{p \neq q} C(p, q)$. Estimating $S_{2}, S_{3}$ and $S_{4}$ in the same way yields

$$
E_{1} \leq C_{1} \cdot \sum_{p \neq q}\left(\mathrm{~V}^{p}(0)+\mathrm{Q}^{p}(j-1)\right)\left(\mathrm{V}^{q}(0)+\mathrm{Q}^{q}(j-1)\right) .
$$

The quantity $E_{2}$ in (23) can be estimated similarly. From (23) we thus have

$$
\mathrm{Q}^{k}(j) \leq 2 C_{1} \cdot \sum_{p \neq q}\left(\mathrm{~V}^{p}(0)+\mathrm{Q}^{p}(j-1)\right)\left(\mathrm{V}^{q}(0)+\mathrm{Q}^{q}(j-1)\right) .
$$


We define,

$$
\mathbf{V}(j)=\sum_{k=1}^{n} \mathrm{~V}^{k}(j), \quad \mathbf{Q}(j)=\sum_{k=1}^{n} \mathrm{Q}^{k}(j),
$$

From (21) and (29)-(30) we conclude that

$$
\mathbf{V}(j) \leq \mathbf{V}(0)+\mathbf{Q}(j-1), \quad \mathbf{Q}(j) \leq C_{2} \cdot(\mathbf{V}(0)+\mathbf{Q}(j-1))^{2},
$$

where $C_{2}=2 n \cdot C_{1}$. It follows that, if the total amount of waves $\mathbf{V}(0)$ in the initial data is sufficiently small, then $\mathbf{Q}(j)$, and hence also the total amount of waves $\mathbf{V}(j)$, remains small for all time steps.

\section{$5 \quad \mathbf{L}^{1}$ Stability}

Following [3], [6] we prove $\mathrm{L}^{1}$ stability by a linearization argument. Consider two initial data $\bar{u}, \bar{v}$ which we join by the path defined by

$$
\theta \mapsto \bar{u}^{\theta}=\theta \bar{u}+(1-\theta) \bar{v}, \quad \theta \in[0,1] .
$$

Let $u_{i, j}^{\theta}$ denote the approximate solution computed with the Godunov scheme applied to the initial data $\bar{u}^{\theta}$ and let $u_{i, j}=u_{i, j}^{1}, v_{i, j}=u_{i, j}^{0}$.

Suppose the left and right states of a Riemann problem depends on $\theta, u_{-}=$ $u_{-}^{\theta}, u_{+}=u_{+}^{\theta}$. The resulting state $\tilde{u}=\tilde{u}^{\theta}$ is given by

$$
\tilde{u}^{\theta}=u_{+}^{\theta}-\left[f\left(u_{+}^{\theta}\right)-f\left(u_{-}^{\theta}\right)\right] .
$$

Differentiation with respect to $\theta$ shows that the infinitesimal perturbations

$$
v_{-}^{\theta}:=\frac{d u_{-}^{\theta}}{d \theta}, \quad v_{+}^{\theta}:=\frac{d u_{+}^{\theta}}{d \theta}, \quad \tilde{v}^{\theta}:=\frac{d \tilde{u}^{\theta}}{d \theta}
$$

satisfy the linearized equation

$$
\tilde{v}^{\theta}=v_{+}^{\theta}-\left[A\left(u_{+}^{\theta}\right) v_{+}^{\theta}-A\left(u_{-}^{\theta}\right) v_{-}^{\theta}\right] .
$$

We decompose the perturbations along the eigenvector bases,

$$
v_{-}^{\theta}=\sum_{k=1}^{n} \nu_{-}^{\theta, k} r^{k}\left(u_{-}^{\theta}\right), \quad v_{+}^{\theta}=\sum_{k=1}^{n} \nu_{+}^{\theta, k} r^{k}\left(u_{+}^{\theta}\right), \quad \tilde{v}^{\theta}=\sum_{k=1}^{n} \tilde{\nu}^{\theta, k} r^{k}\left(\tilde{u}^{\theta}\right),
$$

Letting $\sigma^{\theta}=\left(\sigma^{\theta, 1}, \ldots, \sigma^{\theta, n}\right)$ denote the strengths of the waves in the solution of the Riemann problem $\left(u_{-}^{\theta}, u_{+}^{\theta}\right)$ we observe that the components $\tilde{\nu}^{\theta, k}$ are uniquely determined by $u_{-}^{\theta}, \sigma^{\theta}$ and $\nu_{ \pm}^{\theta}=\left(\nu_{ \pm}^{\theta, 1}, \ldots, \nu_{ \pm}^{\theta, n}\right)$. In analogy with the maps $\Psi^{k}$ above we now define the maps $\Phi^{k}, k=1, \ldots, n$, by

$$
\Phi^{k}\left(u_{-}^{\theta}, \sigma^{\theta}, \nu_{-}^{\theta}, \nu_{+}^{\theta}\right)=\tilde{\nu}^{\theta, k}-\left(\lambda\left(u_{-}^{\theta}\right) \nu_{-}^{\theta, k}+\left[1-\lambda\left(u_{+}^{\theta}\right)\right] \nu_{+}^{\theta, k}\right) .
$$

It is easily shown that

$$
\Phi^{k}\left(u_{-}, \sigma, 0,0\right) \equiv 0, \quad \Phi^{k}\left(u_{-}, 0, \nu_{-}, \nu_{+}\right) \equiv 0 .
$$


It follows that $\Psi^{k}$ admits a representation of the form

$$
\Phi^{k}\left(u_{-}, \sigma, \nu_{-}, \nu_{+}\right)=\sum_{1 \leq p, q \leq n}\left[A_{p, q}^{k} \sigma^{p} \nu_{-}^{q}+B_{p, q}^{k} \sigma^{p} \nu_{+}^{q}\right]
$$

where $A_{p, q}^{k}$ and $B_{p, q}^{k}$ are smooth functions of $u_{-}, \sigma, \nu_{-}, \nu_{+}$. Returning to the scheme we thus have the following system of equations for the components $\nu_{i, j}^{\theta, k}$ of the tangent vectors $d u_{i, j}^{\theta} / d \theta$,

$$
\nu_{i, j+1}^{\theta, k}=\lambda^{k}\left(u_{i-1, j}^{\theta}\right) \nu_{i-1, j}^{\theta, k}+\left[1-\lambda^{k}\left(u_{i, j}^{\theta}\right)\right] \nu_{i, j}^{\theta, k}+\mathcal{Q}_{i, j}^{\theta, k},
$$

where the coupling terms are of the form (35). The $\mathrm{L}^{1}$ norm of the difference between $u_{i, j}=\left.u_{i, j}^{\theta}\right|_{\theta=1}$ and $v_{i, j}=\left.v_{i, j}^{\theta}\right|_{\theta=0}$ at time step $(j+1)$ is now given by

$$
\begin{aligned}
\|u(j+1)-v(j+1)\|_{\mathrm{L}^{1}} & =\sum_{i=-\infty}^{+\infty}\left|u_{i, j+1}-v_{i, j+1}\right| \Delta x \\
& \leq \Delta x \cdot\left(\int_{0}^{1} \sum_{i=-\infty}^{+\infty} \sum_{k=1}^{n}\left|\nu_{i, j+1}^{\theta, k}\right| d \theta\right) \\
& =: \Delta x \cdot \mathcal{N}(j+1) .
\end{aligned}
$$

Using (36) we have

$$
\mathcal{N}(j+1) \leq \mathcal{N}(0)+\int_{0}^{1} \sum_{i=-\infty}^{+\infty} \sum_{k=1}^{n} \sum_{s=0}^{j}\left|\mathcal{Q}_{i, s}^{\theta, k}\right| d \theta
$$

A simple estimate now shows that for a suitable constant $C_{3}$,

$$
\int_{0}^{1} \sum_{i=-\infty}^{+\infty} \sum_{k=1}^{n} \sum_{s=0}^{j}\left|\mathcal{Q}_{i, s}^{\theta, k}\right| d \theta \leq C_{3} \cdot\left(\max _{0 \leq s \leq j} \max _{0 \leq \theta \leq 1} \mathbf{V}^{\theta}(s) \cdot \mathcal{N}(s)\right),
$$

where $\mathbf{V}^{\theta}(s)$ is defined by (30). From above it follows that, if the total amount of waves in the data $\bar{u}, \bar{v}$ are sufficiently small, then $\mathbf{V}^{\theta}(s)$ is uniformly bounded and small. Thus, for data with sufficiently small total variation we have

$$
C_{4}:=C_{3} \cdot\left(\max _{0 \leq s \leq j} \max _{0 \leq \theta \leq 1} \mathbf{V}^{\theta}(s)\right)<1,
$$

such that

$$
\mathcal{N}(j+1) \leq \mathcal{N}(0)+C_{4} \cdot \max _{0 \leq s \leq j} \mathcal{N}(s) .
$$

From (37) we conclude that there exists a Lipschitz constant $L$ such that

$$
\|u(j)-v(j)\|_{\mathrm{L}^{1}} \leq L \cdot\|u(0)-v(0)\|_{\mathrm{L}^{1}} .
$$




\section{Convergence of the Godunov Scheme}

To establish the convergence of the scheme we argue as in [3]. We define a semigroup on $\mathcal{D}$ by using the approximate solutions $u_{\Delta x}$ defined by the Godunov scheme. Given $\bar{u} \in \mathcal{D}$ we approximate it by functions $\bar{u}_{\mu}$ that are piecewise constant on a sequence of grids with mesh tending to zero. Let $u_{\mu}$ denote the corresponding Godunov approximation. By the stability result it follows that, for some subsequence $\mu \rightarrow 0$, there exists the limit

$$
S_{t} \bar{u}:=v(\cdot, t)=\mathrm{L}_{l o c}^{1}-\lim _{\mu \rightarrow \infty} u_{\mu}(\cdot, t)
$$

and this limit defines a uniformly Lipschitz semigroup. Finally, to show that the uniqueness of the limit, by Corollary 9.2 in [5], it suffices to check that the Godunov scheme provides the correct solution locally in time, in case of an initial data $\bar{u}$ which is piecewise constant with simple jumps. That means, we can assume that at each point $x$ where $\bar{u}$ has a jump, the corresponding Riemann problem is solved by waves of one single family. In this case, we are thus dealing essentially with a scalar problem, and the result is well known. Indeed, the Godunov scheme converges to the unique entropy solution when applied to scalar conservation laws. This completes our sketch of the proof of convergence of Godunov approximations to a unique limit. For further details we refer to [9].

Acknowledgement. The research was supported by the European TMR Network on Hyperbolic Systems of Conservation Laws, ERBFMRXCT960033.

\section{References}

[1] M. Arora, P. L. Roe, On postshock oscillations due to shock capturing schemes in unsteady flows, J. Comput. Phys. 130, (1997), 25-40.

[2] S. Bianchini, On a Glimm type functional for a special Jin-Xin relaxation model, Ann. Inst. Henri Poincaré - Nonlinear Analysis, to appear.

[3] S. Bianchini, A. Bressan, BV solutions for a class of viscous hyperbolic systems, Indiana Univ. Math. J., to appear.

[4] A. Bressan, The unique limit of the Glimm scheme, Arch. Rational Mech. Anal. 130, (1995), 205-230.

[5] A. Bressan, Hyperbolic Systems of Conservation Laws. The One Dimensional Cauchy Problem, Oxford University Press, 2000.

[6] A. Bressan, R. M. Colombo, The semigroup generated by $2 \times 2$ conservation laws, Arch. Rational Mech. Anal. 133, (1995), 1-75.

[7] A. Bressan, G. Crasta, B. Piccoli, Well posedness of the Cauchy problem for $n \times n$ systems of conservation laws, Amer. Math. Soc. Memoir, to appear. 
[8] A. Bressan, T. P. Liu, T. YAng, $\mathrm{L}^{1}$ stability estimates for $n \times n$ conservation laws, Arch. Rational Mech. Anal. 149, (1999), 1-22.

[9] A. Bressan, H. K. Jenssen, On the convergence of Godunov scheme for nonlinear hyperbolic systems, Chinese Ann. Math., 21, (2000), 1-16.

[10] A. Bressan, W. Shen, BV estimates for multicomponent chromatography with relaxation, Discrete Cont. Dyn. Syst., 6, (2000), 21-38.

[11] R. J. DiPerna, Convergence of approximate solutions to conservation laws, Arch. Rational Mech. Anal. 82, (1983), 27-70.

[12] J. GLimm, Solutions in the Large for Nonlinear Hyperbolic Systems of Equations, Comm. Pure. Appl. Math. 18 (1965), 697-715.

[13] J. Goodman, Z. Xin, Viscous limits for piecewise smooth solutions to systems of conservation laws, Arch. Rational Mech. Anal. 121, (1992), 235-265.

[14] D. KRöner, Numerical Schemes for Conservation Laws, Wiley and Teubner, 1997.

[15] D. SerRe, Solutions with bounded variations for some hyperbolic systems of conservation laws, J. Differential Equations 68 (1987), 137-168.

[16] S. H. YU, Zero dissipation limit to solutions with shocks for systems of hyperbolic conservation laws, Arch. Rational Mech. Anal. 146, (1999), $275-370$. 